\title{
An FCM-based Personalized Affective Model for Agile Software Development
}

\author{
Wenjing $\mathrm{He}^{1,2}$, Jun $\mathrm{Lin}^{3,4}$, Xinjia $\mathrm{Yu}^{3}$, Zhiqi Shen ${ }^{3}$, Chunyan Miao ${ }^{3}$ \\ ${ }^{1}$ Institute of Computing Technology, Chinese Academy of Sciences, China \\ ${ }^{2}$ University of Chinese Academy of Sciences, China \\ ${ }^{3}$ Joint NTU-UBC Research Centre of Excellence in Active Living for the Elderly (LILY) \\ Nanyang Technological University, Singapore \\ ${ }^{4}$ College of Software, Beihang University, Beijing, China \\ hewenjing@ict.ac.cn, \{junlin, xyu009, zqshen, ascymiao\}@ntu.edu.sg
}

\begin{abstract}
Developers' emotional stability in agile software development (ASD) teams is an important factor affecting the success of a project. Traditional Happiness Chart depends on people's self-report and is unable to produce predictive analysis to facilitate decision-making. In this paper, we proposed a Fuzzy Cognitive Map (FCM) based method for analyzing ASD developers' emotional stability. We designed an easy-touse survey questionnaire which can help establish the values of the causal relationship weights to support personalization of the proposed FCM model. The FCM affective model produces two metrics for assessing a given user's emotional stability: 1) the stable state mood value, and 2) the number of iteration steps for the FCM model to reach equilibrium. The two metrics are evaluated through a real-world experiment involving 20 undergraduate students. The results show that they are strong explanation factors for a user's emotional stability with $99 \%$ confidence. A regression model based on the user study has also been presented in the paper to help researchers study the relationships between the two metrics and a person's emotional stability computationally in the future.
\end{abstract}

\section{INTRODUCTION}

Human factors are important to the success of agile software development (ASD) [1], [2]. ASD team members, especially programmers, are not very keen on expressing their emotions. Nevertheless, team members have emotions. During the development process, their emotional states can be affected by many factors, such as work expectation, delays, quality of software artifacts, etc. The changes in emotion, in return, further affect the software development processes and the results.

In real-world projects, practitioners are starting to take note of this and attempt to use the Happiness Chart (http://agiletrail.com/2011/09/12/ how-to-track-the-teams-mood-with-a-niko-niko-calendar/) to monitor team members' daily mood and encourage emotional openness within the team. An obvious issue with this approach is that it is difficult to know whether one developer's mood will be influenced by the emotions written down by another developer. Other important aspects include accuracy, authenticity, and congruence etc. are also not considered in the method.

In this paper, we investigate the impact of ASD team members' mood stability which has been found to be important for learning, task performance [3] and building trust
[4], [5], [6]. We propose a Fuzzy Cognitive Map (FCM) based method of mood analysis. To enable personalization of the proposed FCM model, we also propose an easy-to-use survey questionnaire which can help establish the values of the FCM causal relationship weights. The FCM affective model is capable of computing two metrics for a given user: 1) the stable state mood value in the range of $[0,1]$ (with 0 indicating very low mood and 1 indicating very high mood) and 2) the number of iterations for the FCM model to reach equilibrium.

To evaluate the explaining power of the two metrics of the proposed FCM affective model, we conducted the a real-world experiment involving 20 undergraduate students formed into ASD teams in Beihang university, China. The results show that the two metrics are strong explanation factors for a user's emotional stability with $99 \%$ confidence. A regression model based on the user study has also been presented in the paper to help researchers study the relationships between the two metrics and a person's emotional stability computationally. The proposed method is a promising approach for analyzing ASD team members' moods.

\section{RELATED WORK}

Emotions in human communication process are very important for perception, decision-making, interaction, and intelligence. Modelling and analyzing ASD team members' mood is essential to reducing the impact of developers' mood swings on the development process. However, there is few research work currently focused on this direction. In this section, we first discuss the general research field of affective computing, and then focus on affective modelling in ASD.

\section{A. Affective Computation Models}

The Ortony, Clore and Collins (OCC) model [7] is the most widely adopted affective model. It regards emotions as the results of the following three types of subjective appraisals:

1) The pleasantness of the consequences of events with respect to the agent's goals: emotions that we call goalbased or event-driven emotions will be stimulated by valenced reactions to consequences of events and appraised by agents' internal goals, including "happy-for", "resentment", "gloating pity", "hope", "fear", "satisfaction", 
"fears-confirmed", "relief”, “disappointment", “joy” and "distress";

2) The approval of the actions by the agent itself or another agent with respect to a set of behavior standards: emotions that we call standard-based or agent-driven emotions will be stimulated by valenced reactions to actions of agents and appraised by agents' internal standards, including "pride", "shame", "admiration" and "reproach";

3) The liking of objects with respect to the attitudes of the agent: emotions that we call attitude-based or objectdriven emotions will be stimulated by valenced reactions to aspects of objects and appraised by agent's internal attitudes, including "love" and "hate".

In general, emotions are affected by both internal and external factors. Internal factors include goals, preferences and attitudes, whereas external factors include events, conditions and other objects in the environment.

Based on the OCC theory, a probabilistic model that assesses student emotions during a game was proposed in [8]. The model can predict a player's emotional state by assessing the player's appraisal of interactions with the game, in light of the player's goals and personality. In [9], an emotional agent was proposed for a serious game based on the Goal Net model [10]. The agent's emotions are modeled by the OCC model powered by an FCM inference engine. Composite emotions from players of a game have also been studied in [11], [12], [13], [14], [15], [16]. The results provide researchers with regression models to infer player emotions based on their observed behaviour in a competitive game environment.

\section{B. Emotion Models in Agile Software Engineering}

An ASD team consists of different roles and people's emotions may significantly affect the outcome of their activities, including those activities in the software development process. The team leader knows that the emotional status of members will affect their tasks and artifacts, but human's emotions and their transition are too complex to observe and control [17].

In [18], the authors attempted to use the affect grid psychological tool to characterize emotions in software requirement engineering. Their results revealed that emotions were key issues in software development activities, and the importance of emotion management in software development life-cycle is important as software development is a human activity. Knowing the emotional state of the development team helps the project manager to create a conducive environment or implement suitable incentives for the team to avoid the effects of undesirable emotions. However, there is current a lack of computational affective models suitable for use in ASD.

\section{PRELIMINARIES}

According to the OCC theory, we know that a developers' mood or emotion is affected by specific events, conditions or other situations in the ASD process. Therefore, we want to build an affective model for ASD team members to simulate interactions between development activites and developers' moods. During our survey, we found modelling and computational capabilities of Fuzzy Cognitive Maps (FCMs) are suitable for building a causal relationship model for the ASD process. This may provide the agile team the ability of viewing emotional state transitions and changes during the process. In this section, we will first introduce the basics of FCMs.

An FCM is a fuzzy-graph structure for causal reasoning. It was developed from the concept of a Cognitive Map (CM) [19]. CMs were used to model a system with concepts and cause-effect relationships. The relationships can be divided into three types: 1) positive, 2) negative, or 3) neutral. CMs can be drawn as a directed graph, the nodes corresponding to relevant concepts/variables in the given domain, and the directed edges denote the relationships between two concepts/variables. The type of a relationship is denoted by a sign associated with the edge. A positive sign indicates a positive relationship with a promoting effect. This means that an increase in the value of the start concept value will lead to an increase in the value of the end concept. Similarly, a negative sign means a negative type with the inhibiting effect. This means an increase in the value of the start concept leads to a decrease in the value of the end concept. No connection between two concepts means that the two concepts are independent from each other.

CMs have two main drawbacks: 1) they do not allow feedbacks; 2) the relationships are binary. Therefore, the use of cognitive maps to model complex systems is infeasible. To tackle them, the Fuzzy Cognitive Maps (FCMs) were proposed as an extension. Compared to CMs, FCMs have two significant enhancements:

1) Causal relationships between concept nodes in an FCM are fuzzified. This character enriches the description of the links by numerical value instead of only using positive, negative and no connection. It allows various degrees to denote different causal influences.

2) FCMs are models for expressing dynamic systems. FCMs can evolve with time, and allows feedback mechanisms. Specifically, the effect of changing the value of one concept node in the model may change the values of other concept nodes, and the change could loop back to the original node in subsequent time steps.

The strength of relationship between two FCM nodes takes on any value in the $[-1,1]$ range. A value of -1 represents the fully negative causal effect, whereas +1 means a fully positive causal effect. Zero denotes no causal effect. Other values denote different fuzzy levels of causal effect. The knowledge of system relationships can be described by a matrix, called the connection matrix. Each cell of this matrix stores a value of a corresponding relationship. The commonly used convention is to place the start nodes in rows and the end nodes in columns.

For example, considering a system with $N$ concept nodes, we have an $N \times N$ matrix representing the FCM. The elements in the matrix are the strengths of the causal relationships. Any one state of the system can be determined by one state vector, which specifies the current values of all system concept nodes. The FCM iteratively updates the state of the system. In one 
iteration, the value of each node is calculated based on the current values of every node exerting influence on it through its causal relationship. After multiplying these values by the edge weight between the two nodes, the sum of these products is taken as the input to a transformation function, which is used to reduce the unbounded inputs to a certain range.

The value of each node in any iteration is computed from values of nodes in the preceding state, using the following equation:

$$
N_{j}(k+1)=f\left(\sum_{i=1}^{n} e_{i j} N_{j}(k)\right)
$$

where $N_{i}(k)$ is the value of the $i$ th node in the $k$ th iteration; $e_{i j}$ is the edge weight between nodes $N_{i}$ and $N_{j} ; n$ is the number of concepts in the FCM; and $f(\cdot)$ is the transformation function. Three types of transformation functions are commonly used. They are the binary, trivalent and sigmoid functions [20]:

1) Bivalent function

$$
f\left(N_{i}(k)\right)= \begin{cases}1 & , \quad N_{i}(k) \leq 0 \\ 0 & , \quad N_{i}(k)>0\end{cases}
$$

2) Trivalent function

$$
f\left(N_{i}(k)\right)= \begin{cases}-1 & , \quad N_{i}(k) \leq-0.5 \\ 0 & , \quad-0.5<N_{i}(k)<0.5 \\ 1 & , \quad N_{i}(k) \geq 0.5\end{cases}
$$

3) Sigmoid function

$$
f\left(N_{i}(k)\right)=\frac{1}{1+e^{-c N_{i}(k)}}
$$

The final results of a simulation performed with FCM strongly depend on the transformation function. If we use the function which results in binary values, the simulation of a FCM system leads to either fixed state pattern of node values (which is called hidden pattern or fixed-point attractor) or a cycling between several states (which is known as the limit cycle). If we use a continuous-output transformation function, the simulation may result in a different outcome. The system may continue to produce different state vector values for successive cycles. In this case, this unstable situation is called a chaotic attractor [21].

The FCM theory has been applied in many domains for more than two decades to model systems or environments with complex causal relationships. These domains include software project management [21], software quality risk analysis [22], software education [23] and agile software development [24].

\section{The Proposed Method}

\section{A. Method}

As FCM theory and method can be used to build causal relationship models for complex systems or processes, we will use it to build affective models for ASD teams, and then use their computational functionality to simulate the interactions between development activities and human moods.

According to OCC theory, external events or activities can affect developers' emotion or mood. Therefore, events (and event results) and developers' mood will be incorporated into the proposed model. Our method includes the following steps:

- Step 1: The team manager designs a general emotional FCMs model for all team members. The concept nodes will include developers' mood, external events/activities and their results. There are two ways to determine the weights between concepts: 1) self-reports by members, and 2) based on members' past behaviour records.

- Step 2: The system executes the FCMs to reach the equilibrium state for each team member. The final state can be treated as a developer's personalized mood level, and the time to reach the state can be used to assess the developer's capability for mood control.

- Step 3: The system monitors the changes at the early stage (when the node values change rapidly), and how many iterations the model takes to reach the equilibrium state for each individual.

- Step 4: The system shows a comparison score list to help the team manager predict who will be the most suitable candidate to handle a given ASD activity.

To simplify the model, we consider their complex personal emotions as one comprehensive mood factor. According to common ASD experience and ignoring other factors, the causal relationships between developer's mood status, progress of task execution and quality of task execution during development process are shown in the FCMs in Figure 1(a). The general model presents the relationships that are essential during an ASD process, which consists of three concept nodes as follows:

- C1: Worker's Mood Status (called "Mood" for short) reflects the comprehensive feelings to an event or an event result. The mood value can include "high", "medium" and "low" or finer granularities. They affect or are affected by the results of task executions. The mood values can be acquired by the AffectButton [25] and then normalized in a range of $[0,1]$.

- C2: Task Execution Progress (called "Progress" for short) can be interpreted as a relative comparison concept for the task execution progress. Its value can be calculated by the ratio of actual task completed time to estimated task completed time, and then normalized into a range of $[0,1]$.

- C3: Task Execution Quality (called "Quality" for short) can be interpreted as a relative comparison concept for artifact quality after task execution. Its value can be acquired through a rating assessment by other team members, and then normalized into a range of $[0,1]$.

The casual relationships between nodes are represented by directed edges with fuzzy weights based on human experience acquired through questionnaires.

\section{B. Illustrative Examples}

To illustrative the proposed method, we look into two fictitious developers, "Michael" and "Grace". From the FCM model (Figures 1(b) and 1(c)), we can see the mood of the 


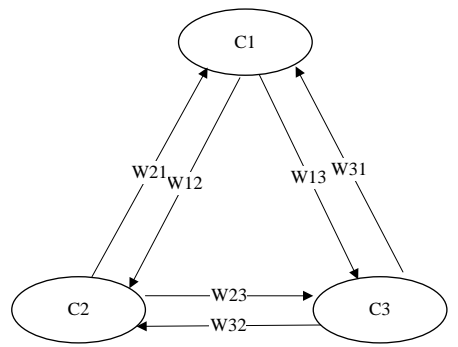

(a) Developer Michael's model

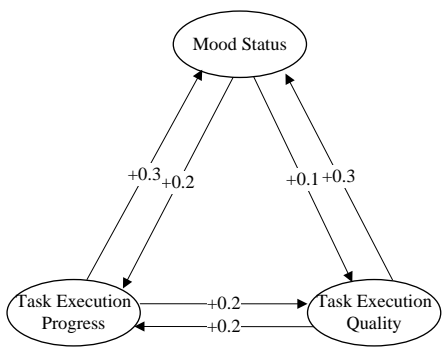

(b) Developer Michael's model

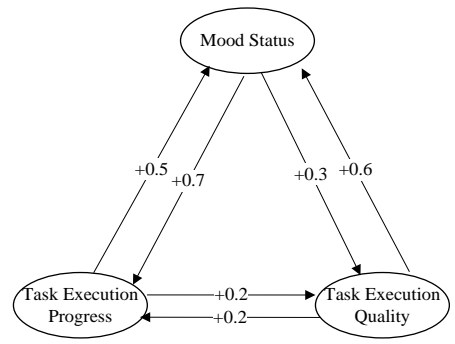

(c) Developer Grace's model

Fig. 1. The proposed general developers' FCM model.

developer who picks up the task has positive effect on the task execution progress (an edge weight of 0.2 from $C 1$ to $C 2$ for Michael; and an edge weight of 0.7 from $C 1$ to $C 2$ for Grace). This means the progress of task execution will be good when the developer's mood status is at a high level. Meanwhile, the task execution progress also positively influences developers' mood (an edge weight of 0.3 from $C 2$ to $C 1$ for Michael; and an edge weight of 0.5 from $C 2$ to $C 1$ for Grace). This means developers mood is low when the task is delayed and vice verse. This conforms to the common sense in real projects.

Furthermore, the developers' mood positively impacts the task execution quality (an edge weight of 0.1 from $C 1$ to $C 3$ for Michael; and an edge weight of 0.3 from $C 1$ to $C 3$ for Grace). On the other hand, the increase in quality can lead to higher mood among the developers (an edge weight of 0.3 from $C 3$ to $C 1$ for Michael; and an edge weight of 0.6 from $C 3$ to $C 1$ for Grace). Generally, good task execution progress also leads to high quality (an edge weight of 0.2 from $C 2$ to $C 3$ for Michael; and an edge weight of 0.2 from $C 2$ to $C 3$ for Grace). High quality software with fewer bugs will make the overall progress faster (an edge weight of 0.2 from $C 3$ to $C 2$ for Michael; and an edge weight of 0.2 from $C 3$ to $C 2$ for Grace). The strengths of the relationships were established based the developers' personal experience.

The FCM-based model for Michael can be presented in a matrix:

$$
\left[\begin{array}{ccc}
0 & 0.2 & 0.1 \\
0.3 & 0 & 0.2 \\
0.3 & 0.2 & 0
\end{array}\right] .
$$

The matrix for Grace's FCM model:

$$
\left[\begin{array}{ccc}
0 & 0.7 & 0.3 \\
0.5 & 0 & 0.2 \\
0.6 & 0.2 & 0
\end{array}\right] .
$$

Next, simulations are run based on the FCM models. The starting vector is denoted as Iteration \#0. Each state vector consists of three numbers, which correspond to the conceptual nodes Mood (C1), Progress (C2), and Quality $(C 3)$. The simulation begins with the start state vector Iteration $\# 0=(0.5,0,0)$ for both developers, which represents a situation in which Mood is active and set at a medium value of 0.5 , and other concepts are inactive (i.e. the developer has not yet started to work). As the simulation continues, successive values of the nodes show trends which occur with the progressing time. By analyzing the states of the nodes in the simulation process, observations obtained and analyzed.

The simulation was carried out using the logistic sigmoid function as a threshold function, which is a continuousoutput transformation function and thus provides true fuzzy conceptual node states. We mainly want to see the comparative results between two developers, so the constant $c$ can be set to a common number 5 .

For Michael, the model reaches equilibrium at Iteration $\# 14=(0.92058,0.846519,0.786979)$. For Grace, the model reaches equilibrium at Iteration $\# 7=(0.994717,0.987922,0.922728)$. Figure 2 shows the results of Michael's model in a plot, and Figure 3 shows the results of Grace's model in a plot.

This model describes state change trends in a task development activity. It characterizes a situation when a worker starts to do a task. Successive states of the modeled system show changes in concept node values, which represent workers' mood and task execution aspects. The final state achieved by the model shows concept nodes in equilibrium states. This can be considered as the prediction by the model on the likely outcomes of the development activities and the developers' mood.

The first iteration (\#1) shows the beginning of execution of the ASD activities. As time goes by, the workers' mood and quality of work increase rapidly at first. At iteration $\# 1$, the workers need some time to familiarize with the new task and environment. Therefore, their progress was not fast and mood

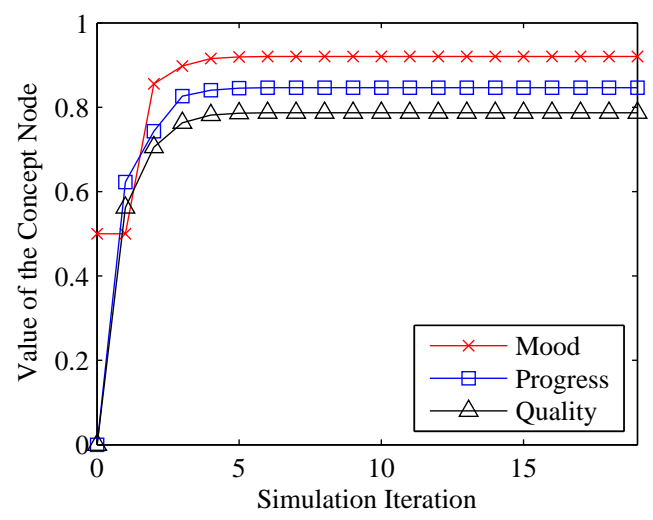

Fig. 2. Results of Michael's first model. 


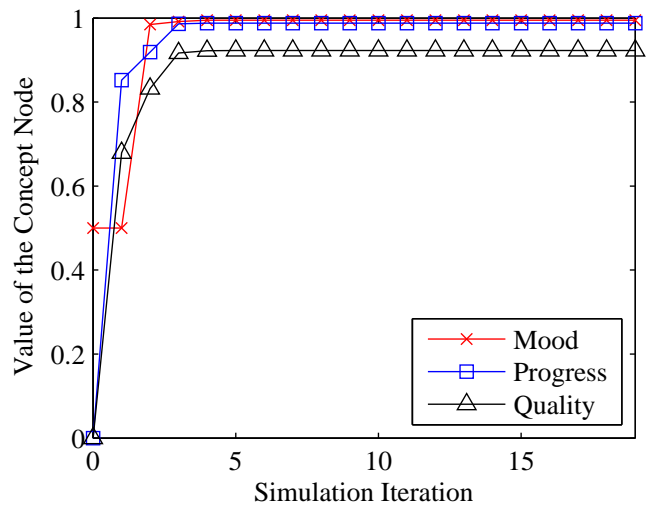

Fig. 3. Results of Grace's first model.

is not high. After this period, the mood value increases and then stays at a stable level, especially for Grace. For Michael, after the quality reaching the highest value at Iteration \#12 and the progress reaching the highest value at Iteration \#11, the mood reached the equilibrium value at iteration \#14. For Grace, before the quality reaching the highest at Iteration \#7, the mood and the progress both reach the highest values at Iteration \#6 and then stay at the equilibrium. This result shows that the Grace needs less time to reach the stable state, and her final state performs better than Michael.

\section{EMPIRICAL EVALUATION}

We have engaged 20 undergraduate software engineering students taking the ASD course in our user study. Each student completed the questionnaire so that the proposed model can be personalized to each of them. The students form into 4 ASD teams of 5 people each to work on coursework projects spanning over 8 weeks. We have interviewed the course instructors about the students' emotional stability characteristics based on their observations throughout the coursework projects. They provided ratings on each student $i$ 's emotional stability, $E S_{i}$, on an 11-point Likert scale [26] (0-10 with 0 representing "very poor emotional stability" and 10 representing "very good emotional stability"). The instructor provided ratings are treated as the ground truth in this user study. The distribution of the 20 students' stable state mood values against the number of steps taken to reach equilibrium as calculated by the proposed FCM model is shown in Figure 4(a).

Let $i$ denote a student. Firstly, we study the correlation between the students' stable state mood $\left(M_{i}\right)$ and the number of iteration steps $\left(S_{i}\right)$ taken to reach the equilibrium state according to the proposed FCM model with the instructors' ratings on their emotional stability $\left(E S_{i}\right)$. Figure 4(b) shows the distribution of $M_{i}$ against $E S_{i}$ for all $i$. The correlation coefficient between $M_{i}$ and $E S_{i}$ is 0.6996 , indicating a strong positive correlation. Figure 4(c) shows the distribution of $S_{i}$ against $E S_{i}$ for all $i$. The correlation coefficient between $S_{i}$ and $E S_{i}$ is -0.9383 , indicating a very strong negative correlation. Thus, among the two metrics proposed in this
TABLE I

REgRESSION ANALYSIS RESULTS

\begin{tabular}{crrr}
\hline Constants & Coefficient & Std. Error & \multicolumn{1}{c}{$p$-value } \\
\hline$\alpha_{1}$ & $5.818^{* * *}$ & 1.543 & 0.00152 \\
$\alpha_{2}$ & $-0.753^{* * *}$ & 0.072 & $8.63 \times 10^{-9}$ \\
$\beta$ & $8.781^{* * *}$ & 1.911 & 0.00026 \\
\hline Note: ${ }^{* * *}: p<0.01$. & & \\
\hline
\end{tabular}

study, $S_{i}$ shows stronger explanation power for a person's emotional stability than $E S_{i}$.

Based on the study results, we investigate the following regression model which can be used to estimate a person's emotional stability based on results produced by the proposed FCM method:

$$
E S_{i}=\alpha_{1} M_{i}+\alpha_{2} S_{i}+\beta .
$$

$\alpha_{1}$ and $\alpha_{2}$ are the linear regression coefficients, whereas $\beta$ is the $y$-axis intercept value.

Based on the analysis of the 20 study participants' data, the regression analysis results are shown in Table I. It can be concluded that the stable state mood $M_{i}$ and the equilibrium steps $S_{i}$ of the proposed method can be used as explanatory factors for a student $i$ 's emotional stability $E S_{i}$ in the context of ASD with $99 \%$ confidence.

\section{Vi. CONClusions And Future Work}

In this paper, we proposed a Fuzzy Cognitive Map (FCM) based method for analyzing an important and difficult to measure concept involved in ASD - a developer's emotional stability. We designed an easy-to-use survey questionnaire which can help establish the values of the causal relationship weights to support personalization of the proposed FCM model for each individual user. The FCM affective model is produces two metrics for assessing a given user's emotional stability, which is important to the progress and quality of a software project. They are: 1) the stable state mood value, and 2) the number of iteration steps for the FCM model to reach equilibrium. The two metrics are evaluated through a realworld experiment involving 20 undergraduate students. The results show that the proposed metrics, especially $S_{i}$, are strong explanation factors for a user's emotional stability with 99\% confidence. A regression model based on the user study has also been presented in the paper to help researchers study the relationships between the two metrics and a person's emotional stability computationally.

From this work, we see a series of interesting future research directions. In this work, we are currently using the basic FCM model. However, there are many sophisticated FCM models, such as [27], which helps reduce human intervention at various stages. In subsequent research, we plan to look into these models to improve the proposed FCM affective model. As the current user study involved only 20 subjects who are all undergraduate students, we refrain from generalizing our findings. In the future, we will conduct larger scale empirical 

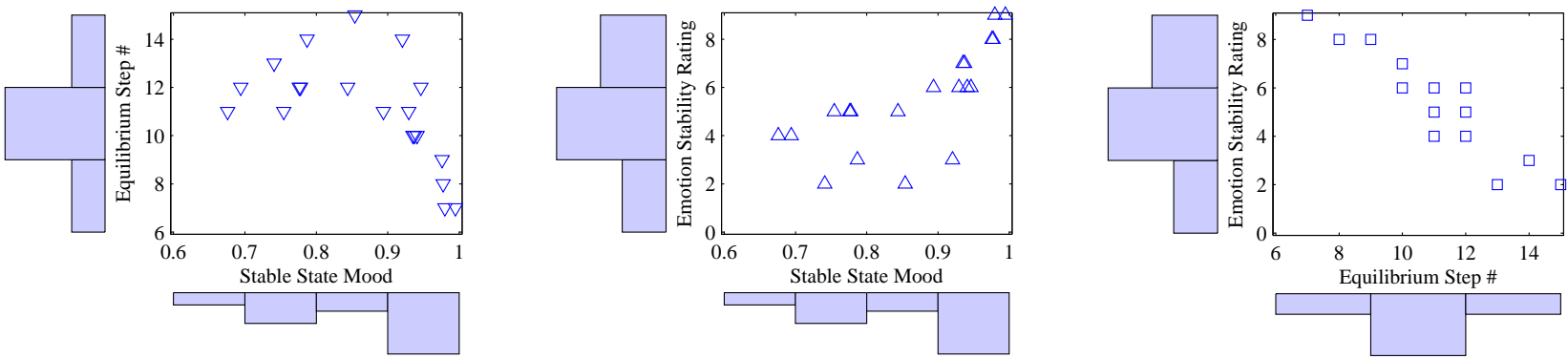

(a) The distribution of students' stable state mood (b) The distribution of students' stable state mood (c) The number of steps taken to reach equilibrium values vs. the number of steps taken to reach values under the proposed FCM model vs. the under the proposed FCM model vs. the instructors' equilibrium under the proposed FCM model. instructors' ratings on their emotional stability. ratings on their emotional stability.

Fig. 4. Empirical Findings.

studies involving subjects with more diverse backgrounds to improve the generalizability of the results obtained.

\section{ACKNOWLEDGEMENTS}

This research is supported by the National Research Foundation, Prime Minister's Office, Singapore under its IDM Futures Funding Initiative.

\section{REFERENCES}

[1] A. Cockburn and J. Highsmith, "Agile software development: The people factor," Computer, vol. 34, no. 11, pp. 131-133, 2001.

[2] J. Lin, H. Yu, Z. Shen, and C. Miao, "Studying task allocation decisions of novice agile teams with data from agile project management tools," in Proceedings of the 29th ACM/IEEE International Conference on Automated Software Engineering (ASE'14), 2014, pp. 689-694.

[3] E. Eldar and Y. Niv, "Interaction between emotional state and learning underlies mood instability," Nature Communications, vol. 6, no. 6149, p. doi:10.1038/ncomms7149, 2014.

[4] Z. Shen, H. Yu, C. Miao, and J. Weng, "Trust-based web-service selection in virtual communities," Journal for Web Intelligence and Agent Systems (WIAS), vol. 9, no. 3, pp. 227-238, 2011.

[5] Y. Liu, S. Liu, H. Fang, J. Zhang, H. Yu, and C. Miao, "Reprev: Mitigating the negative effects of misreported ratings," in Proceedings of the 28th AAAI Conference on Artificial Intelligence (AAAI-14), 2014, pp. 3124-3125.

[6] H. Yu, Z. Shen, C. Miao, B. An, and C. Leung, "Filtering trust opinions through reinforcement learning," Decision Support Systems (DSS), vol. 66, pp. 102-113, 2014.

[7] A. Ortony, G. L. Clore, and A. Collins, The Cognitive Structure of Emotions. Cambridge University Press, 1990.

[8] C. Conati, "Probabilistic assessment of user's emotions in educational games," International Journal of Applied Artificial Intelligence, vol. 16, no. 7-8, pp. 555-575, 2002.

[9] H. Zhang, Z. Shen, X. Tao, C. Miao, B. Li, Ailiya, and Y. Cai, "Emotional agent in serious game (DINO)," in Proceedings of the 8th International Conference on Autonomous Agents and Multiagent Systems (AAMAS'09), 2009, pp. 1385-1386.

[10] J. Lin, H. Yu, Z. Shen, and C. Miao, "Using goal net to model user stories in agile software development," in Proceedings of the 15th IEEE/ACIS International Conference on Software Engineering, Artificial Intelligence, Networking and Parallel/Distributed Computing (SNPD'14), 2014, pp. 1-6.

[11] H. Yu, Z. Shen, C. Miao, and A.-H. Tan, "A simple curious agent to help people be curious," in Proceedings of the 10th International Conference on Autonomous Agents and Multiagent Systems (AAMAS'11), 2011, pp. $1159-1160$.

[12] Q. Wu, X. Han, H. Yu, Z. Shen, and C. Miao, "The innovative application of learning companions in virtual singapura," in Proceedings of the 12th International Conference on Autonomous Agents and Multiagent Systems (AAMAS'13), 2013, pp. 1171-1172.
[13] Y. Cai, Z. Shen, S. Liu, H. Yu, X. Han, J. Ji, M. J. McKeown, C. Leung, and $\mathrm{C}$. Miao, "An agent-based game for the predictive diagnosis of parkinson's disease," in Proceedings of the 13th International Conference on Autonomous Agents and Multiagent Systems (AAMAS'14), 2014, pp. 1663-1664.

[14] J. Lin, H. Yu, C. Miao, and Z. Shen, "An affective agent for studying composite emotions," in Proceedings of the 2015 International Conference on Autonomous Agents and Multiagent Systems (AAMAS'15), 2015, pp. $1947-1948$

[15] X. Yu, C. T. Salmon, and C. Leung, "Emotional interactions between artificial companion agents and the elderly," in Proceedings of the 2015 International Conference on Autonomous Agents and Multiagent Systems (AAMAS'15), 2015, pp. 1991-1992.

[16] X. Yu, C. Miao, C. Leung, and C. T. Salmon, "Modelling composite emotions in affective agents," in Proceedings of the 2015 IEEE/WIC/ACM International Joint Conference on Web Intelligence and Intelligent Agent Technology (WI-IAT'15), 2015.

[17] S. D. Fraser, F. P. Brooks, Jr., M. Fowler, R. Lopez, A. Namioka, L. Northrop, D. L. Parnas, and D. Thomas, ““No silver bullet” reloaded: Retrospective on "Essence and accidents of software engineering"," in Companion to the 22nd ACM SIGPLAN Conference on Objectoriented Programming Systems and Applications Companion (OOPSLA'07), 2007, pp. 1026-1030.

[18] R. Colomo-Palacios, C. Casado-Lumbreras, P. Soto-Acosta, and Ángel Garca-Crespo, "Using the affect grid to measure emotions in software requirements engineering," Journal of Universal Computer Science, vol. 17, no. 9, pp. 1281-1298, 2011

[19] B. Kosko, "Fuzzy cognitive maps," International Journal of ManMachine Studies, vol. 24, no. 1, pp. 65-75, 1986.

[20] A. K. Tsadiras, "Comparing the inference capabilities of binary, trivalent and sigmoid fuzzy cognitive maps," Information Sciences, vol. 178, no. 20, pp. 3880-3894, 2008 .

[21] W. Stach and L. Kurgan, "Modeling software development projects using fuzzy cognitive maps," in Proceedings of the 4th ASERC Workshop on Quantitative and Soft Software Engineering (QSSE'04), 2004, pp. 5560 .

[22] N. Bhatia and N. Kapoor, "Fuzzy cognitive map based approach for software quality risk analysis," ACM SIGSOFT Software Engineering Notes, vol. 36, no. 6, pp. 1-9, 2011

[23] S. Hossain and L. Brooks, "Fuzzy cognitive map modelling educational software adoption," Computer and Education, vol. 51, no. 4, pp. 15691588, 2008.

[24] L. Cao, B. Ramesh, and T. Abdel-Hamid, "Modeling dynamics in agile software development," ACM Transactions on Management Information Systems (TMIS), vol. 1, no. 1, pp. 5:1-5:26, 2010.

[25] J. Broekens and W.-P. Brinkman, "AffectButton: A method for reliable and valid affective self-report," International Journal of HumanComputer Studies, vol. 71, no. 6, pp. 641-667, 2013.

[26] R. Likert, "A technique for the measurement of attitudes," Archives of Psychology, vol. 22, no. 140, pp. 1-55, 1932.

[27] Y. Miao, "Modelling dynamic causal relationship in fuzzy cognitive maps," in Proceedings of the 2014 IEEE International Conference on Fuzzy Systems (FUZZ-IEEE'14), 2014, pp. 1013-1020. 